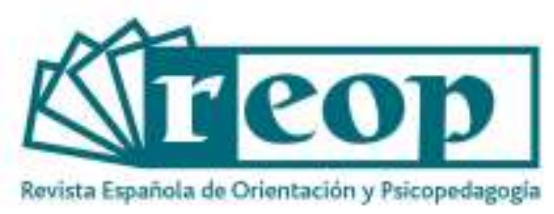

\title{
RELACIÓN ENTRE FAMILIAS MIGRANTES Y PROFESORADO: UN CAMINO POR CONSTRUIR
}

\author{
THE RELATIONSHIP BETWEEN MIGRANT FAMILIES AND FACULTY: A ROAD TO \\ BUILD
}

\author{
Pilar Arnaiz-Sánchez \\ Mohamed Chamseddine Habib Allah ${ }^{1}$ \\ Universidad de Murcia. Facultad de Educación. Departamento de Didáctica y Organización \\ Escolar. Murcia, España
}

\section{RESUMEN}

La relación de las familias migrantes con el profesorado tiene sus propias características y peculiaridades, así como una palpable carencia de orientación psicopedagógica adecuada para fomentar el conocimiento y el reconocimiento mutuo e impulsar de esta manera la desaparición de las diferencias significativas que suelen aflorar entre ambas partes a la hora de afrontar los desafíos de la comunicación. En tal sentido, el objetivo de la investigación abordada en este trabajo, fue describir el conocimiento de la vida del centro que poseen los progenitores migrantes, el conocimiento que posee el profesorado sobre la vida del alumnado migrante y el tipo de relaciones personales que mantienen ambas partes, en centros de educación infantil y primaria de atención preferente de la Región de Murcia. Para llevar a cabo este estudio se empleó un método descriptivo e inferencial, donde se aplicaron dos cuestionarios, uno dirigido a los padres y otro a los docentes. Participaron 823 progenitores y 150 profesores. Los resultados indican que el profesorado apenas conoce personalmente a los padres varones de sus alumnos, y que las visitas de los padres por iniciativa propia continúan siendo una asignatura pendiente. Sin embargo, se aprecian avances relevantes en cuanto a la accesibilidad y predisposición para la comunicación

\footnotetext{
1 Correspondencia: Mohamed Chamseddine Habib Allah, Universidad de Murcia, Departamento de Didáctica y Organización Escolar, Facultad de Educación. Campus de Espinardo. CP: 30100. Ciudad de Murcia, España. Teléfono: 868884 015. Correo electrónico: mohamed.c.h@um.es
} 
por parte de ambas instituciones educativas, si bien queda mucho por hacer en relación a la consolidación de confianza.

Palabras Clave: Familias migrantes, profesorado, orientación psicopedagógica, conocimiento y reconocimiento mutuo

\begin{abstract}
Relationship between teachers and migrant families has its own characteristics and peculiarities, as well as a palpable lack of adequate psycho-pedagogical guidance to foster knowledge and mutual recognition, promoting the disappearance of significant differences that usually emerge between both parties when it comes to facing the communication challenges. In this sense, the research objective undertaken in this assignment was to describe migrant parents' knowledge about life of the Centre, faculty's knowledge about migrant students' life and personal relationships maintained by both parties at preferential pre-school and elementary education Centre's in Region de Murcia. In order to carry out this study, a descriptive and inferential method was used in which two questionnaires were applied, one addressed to parents and the other addressed to teachers. 823 parents and 150 teachers participated. The results indicate that teachers have little personal knowledge about their students' male parents, and parents' visits by their own initiative continue to be a pending subject. However, significant progress has been made in terms of accessibility and readiness for communication on the part of both educational institutions, although much remains to be done in terms of building confidence.
\end{abstract}

Key words: Migrant families, teachers, guidance, mutual knowledge and recognition

\title{
Cómo citar este artículo:
}

Arnaiz Sánchez, P., y Chamseddine Habib Allah, M., (2020). Relación entre familias migrantes y profesorado: un camino por construir. Revista Española de Orientación y Psicopedagogía, 31(2), 115-131. https://doi.org/10.5944/reop.vol.31.num.2.2020.27989

\section{Introducción}

Las relaciones entre las familias y la escuela a veces no fluyen con la sintonía que sería esperable, quizás porque ambas partes se instalan en posturas diferentes, que generan a menudo cierta conflictividad, debido a la relación asimétrica entre los dos colectivos (Rey Mantilla, 2006), lo que ha sido calificado como paz armada por Dubet (1997). Autores como Andrés y Giró (2016) corroboran el nivel de recelo existente entre el profesorado y las familias, lo que influye en el proceso de comunicación entre ambas partes y produce críticas bidireccionales. La escuela culpabiliza a las familias de su falta de conocimiento de las normas y límites establecidos en los centros y, a la inversa, las familias desconfían de la escuela ante sus dificultades para acceder a los canales de participación circunscritos en el marco escolar en igualdad de condiciones.

Si centramos nuestro interés en las familias migrantes y en los docentes, apreciamos que 
dicha relación tiene sus propias características y peculiaridades (Chamseddine y Hernández Martín, 2020). La opinión de los progenitores migrantes sobre la escuela, en general, y sobre el profesorado, en particular, muestra la ausencia de tiempo y las dificultades idiomáticas como las principales causas de la limitada relación que mantienen con el profesorado. A ello añaden las diferencias de trato que los docentes otorgan a las familias autóctonas, frente al que dan a las migrantes, definido por estas como hostil, lejano y frío (Chamseddine, 2020; Jiménez, 2007).

Las propias familias migrantes reconocen su escasa relación con el profesorado, que justifican por el reducido tiempo del que disponen para ir a la escuela ya que sus necesidades económicas les hacen trabajar muchas horas, unido al desempeño de sus quehaceres familiares (Llevot y Bernard, 2015). Por ello, estas familias migrantes reclaman de la escuela una intervención orientadora y psicopedagógica, intencionada, sistemática y programada en la práctica docente, que tenga en cuenta su situación, que escuche sus voces y opiniones, y que facilite canales de comunicación y participación entre ambas instituciones. Esta actuación implicaría beneficios para todos y cada uno de los agentes educativos, y permitiría una intervención orientadora no excluyente en y desde la escuela.

El estudio realizado por González (2007) expresa que los docentes viven con cierta angustia e incertidumbre las situaciones en las que tienen que comunicarse con las familias migrantes, debido a sus limitaciones idiomáticas y a los prejuicios y estereotipos que muchas veces tienen sobre su cultura de origen. Esta situación alimenta el rechazo y el recelo entre ambas partes, dificultando una interacción fluida y posturas de acercamiento e intercambio. No obstante, el profesorado insiste en que los padres y las madres migrantes deben ser conscientes de que la calidad de la educación comienza con su colaboración, contribución e implicación de manera activa en el centro educativo (Ceballos y Saiz, 2019; Macía, 2019; Madridet al., 2019; SantosRego et al, 2019).

Tras una revisión bibliográfica exhaustiva sobre la relación de las familias migrantes con el contexto escolar, se ha comprobado que numerosas investigaciones han considerado el escaso conocimiento de la vida del centro que poseen las familias migrantes, cuestión que obstaculiza no sólo la participación de dichos padres en la dinámica familiar, sino también la inclusión y el éxito escolar de sus hijos e hijas (García Sanz et al., 2010; Gigli et al., 2019; Hernández Prados et al., 2016; Hernández Prados y Alcántara, 2016; Intxausti et al., 2014; Terrén y Carrasco, 2007). Dicho desconocimiento requiere una intervención psicopedagógica desde la escuela que insista en la función orientadora para minimizar el riesgo de exclusión educativa de estos padres, representando sus intereses, necesidades y prioridades, para mejorar la calidad de su relación e interacción con la escuela.

También existen antecedentes en la literatura especializada en esta temática que confirman que el conocimiento que posee el profesorado sobre la vida del alumnado migrante tampoco goza de buenos indicadores, ya que suele ser escasa (Aparicio, 2003; Aguado et al., 2003; Benner y Mistry 2007; Gómez Jarabo; 2015; Intxausti et al., 2014; Leiva, 2013a, 2013b; Rodríguez y Fernández, 2018; Rubie-Davies et al., 2010; Wood et al., 2007). Esta falta de información de diferentes hechos, tanto dentro como fuera de la escuela, puede afectar a la comprensión de los elementos clave que obstaculizan o facilitan el destino académico del alumnado en cuestión.

Si nos adentramos en el estudio de las relaciones que mantienen docentes y progenitores migrantes, se hace latente que la interacción entre las dos partes ha experimentado tímidos avances en los últimos años (Charette y Kalubi, 2016; Fagan, 2013; Hampden y Galindo, 2016; Garreta y Llevot, 2007; Loudová, 2013; Martínez González y Pérez Herrero, 2006; Megías Quirós, 2006; Portes y Rumbaut, 2001; Roth y Volante, 2018; Vallespir et al., 2016). Por ello, estos autores abogan por el desarrollo de relaciones más interactivas en un contexto de asesoramiento y orientación que actúe en red para facilitar la información, la comunicación y la cooperación entre los dos agentes educativos. 
A la vista de lo expresado, el propósito de esta investigación es conocer cómo acontece lo anteriormente expuesto en centros de atención preferente de educación infantil y primaria de la Región de Murcia, denominados así porque a ellos asisten alumnos vulnerables por diferentes causas, centrando la atención en el colectivo de alumnos migrantes. Así, el objetivo general se centra en analizar el conocimiento que poseen las familias migrantes sobre el centro escolar, el conocimiento que tienen los docentes sobre la vida del alumno y el tipo de relaciones que mantienen ambas partes.

- Analizar el conocimiento de la vida del centro que poseen las familias migrantes.

- Describir el conocimiento que posee el profesorado sobre la vida del alumnado migrante.

- Identificar el tipo de relaciones personales que mantienen las familias migrantes, y el profesorado.

\section{Método}

\section{Población y muestra}

En la Región de Murcia existen 15 centros de educación infantil y primaria considerados de atención preferente por parte de Consejería de Educación y Deporte cuyo alumnado reúne cuatro requisitos: a) presenta necesidades de compensación educativa -incorporación tardía, desconocimiento del castellano y desfase curricular de dos o más cursos-; b) precisa ayudas de comedor y para libros; c) tasa de absentismo anual y alumnado repetidor y c) hay profesorado sin destino definitivo.

El muestreo llevado a cabo para la elección de los participantes fue intencional con la consideración de que asistiera a dichos centros alumnado migrante. Contamos con 10 centros educativos, ya que en cuatro no había alumnado migrante, y otro cubría únicamente Educación Secundaria. De una población de 2651 familias del alumnado escolarizado en dichos centros, para un nivel de confianza del $95 \%$ y un $3 \%$ de margen de error, se necesitarían 761 familias. En previsión de una posible muerte muestral, finalmente contamos con una muestra de 832 . La muestra del profesorado se eligió por derivación de las familias, considerando una media de 2 hijos por familia y una ratio de 1 profesor por 25 alumnos para 832 familias. Habríamos necesitado una muestra de 67 profesores, pero quisieron participar 150 profesionales, lo que supone un nivel de confianza del $95 \%$ con un margen de error inferior al $5 \%$.

Seguidamente, se expone el perfil de las familias respecto a su nivel de formación (Figura 1). En lo que se refiere a las madres, la mayor parte de ellas no supera los estudios primarios (287), la E.S.O (258), sin estudios (145), bachillerato o formación profesional (124), y estudios superiores (18). Examinando el nivel de estudios de los padres, aparece un número muy elevado que no supera los estudios primarios (320), la E.S.O (251), bachillerato o formación profesional (126), sin estudios (109), y estudios superiores (26). 


\section{Figura 1}

Nivel de estudio de madres y padres.

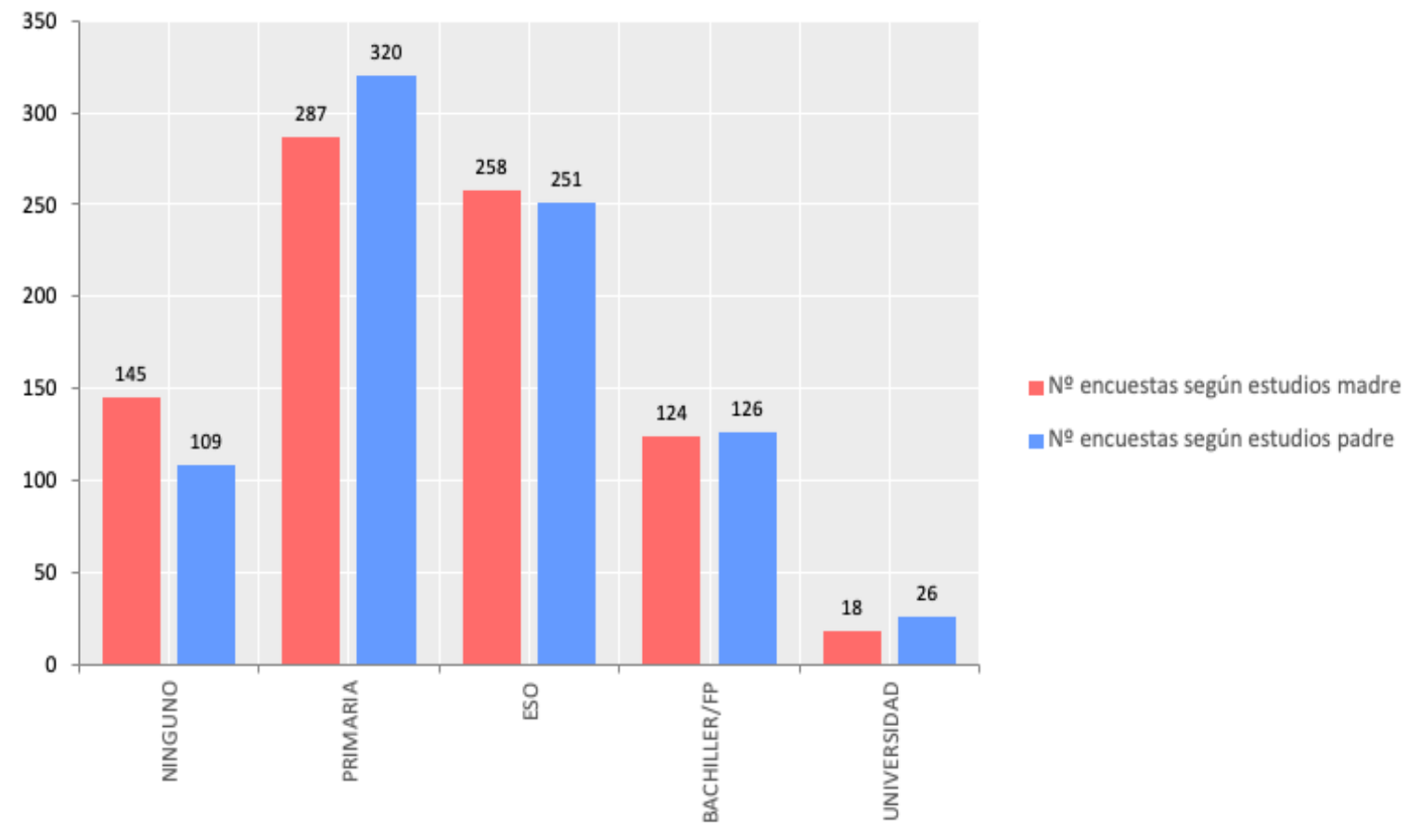

Fuente: Elaboración propia

En cuanto a los 150 docentes participantes (Figura 2), 36 pertenecen a educación infantil (14, 15 y 7 de $1^{\circ} \stackrel{\circ}{2} 2^{\circ}$ y $3^{\circ}$ curso respectivamente) y 114 a educación primaria $\left(16,28,31,12,19\right.$ y 8 , de $1^{\circ}$ a 6 o curso respectivamente).

\section{Figura 2}

El curso del que los profesores participantes.

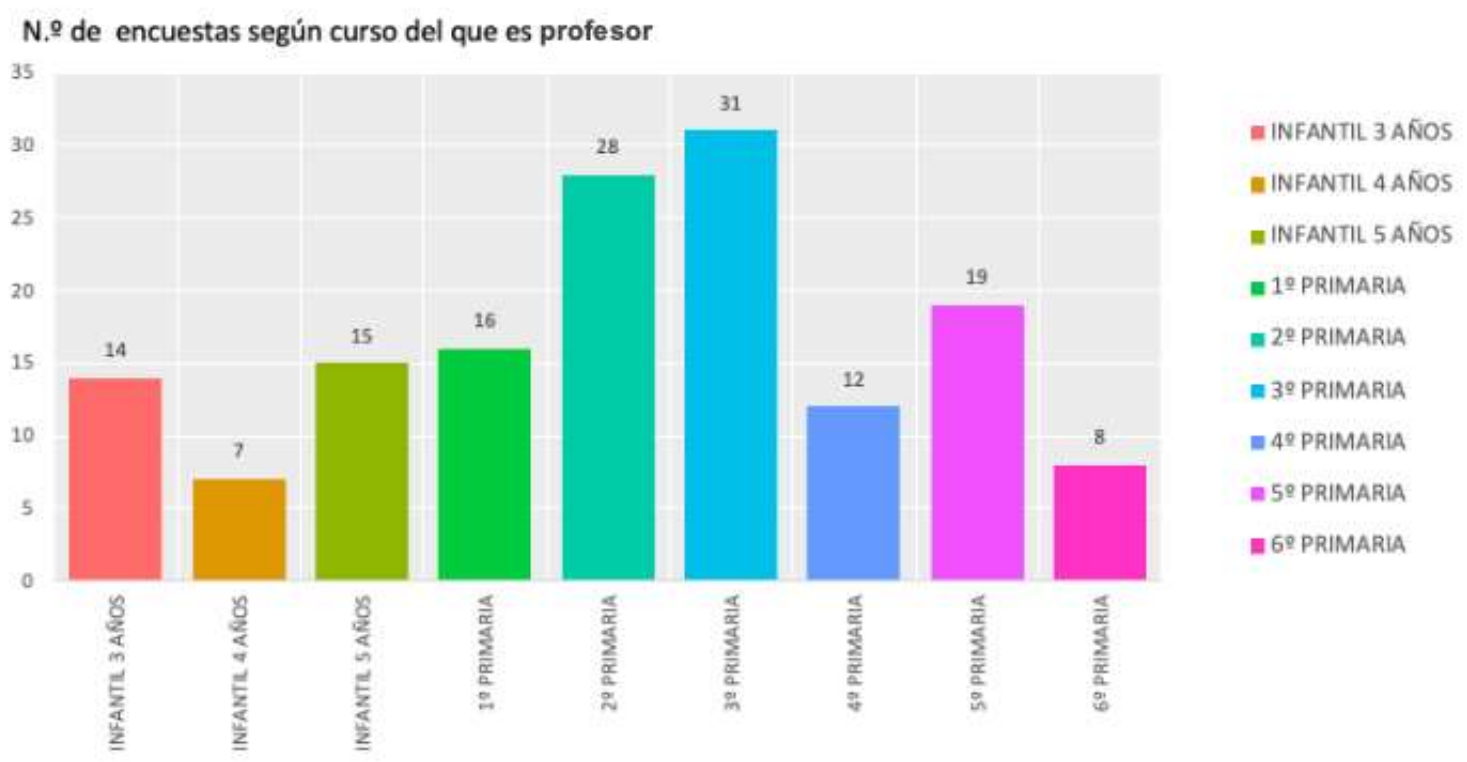

Fuente: Elaboración propia 


\section{Instrumentos}

Los instrumentos utilizados en este estudio han sido dos cuestionarios, cuyos autores son Gomáriz et al. (2008). El primero está destinado a las familias y consta de 49 ítems; el segundo está dirigido al profesorado y consta de 53 ítems. Dichos instrumentos disponen de cinco alternativas de respuesta de escala Likert: nunca (1), pocas veces (2), algunas veces (3), frecuentemente (4) y siempre o casi siempre (5).

En este trabajo se abordaron 5 dimensiones a través de 40 ítems. El cuestionario de las familias consta de 2 dimensiones: conocimiento de la vida del centro ( 7 ítems) y relaciones con los docentes (7 ítems). Por su parte, el cuestionario del profesorado está formado por 3 dimensiones: su percepción sobre el conocimiento del centro por las familias migrantes (7 ítems), su conocimiento sobre la vida del alumno (12 ítems), y tipo de relaciones que mantiene con las familias (7 ítems).

La validez de ambos cuestionarios se obtuvo a través del juicio por expertos, participando 8 ( 3 profesores universitarios y 5 docentes de primaria y secundaria expertos en el tema). La fiabilidad se obtuvo a través de la obtención del Alpha de Cronbach. El cuestionario de las familias alcanzó a nivel global de .911, obteniendo la primera dimensión un valor de .767 y la segunda de .721. La fiabilidad global del instrumento de los profesores es de .825, y la de cada una de las 3 dimensiones que lo componen $.783, .717$ y .525 respectivamente.

\section{Diseño y procedimiento}

Este estudio tiene la finalidad de explicar y pronosticar los fenómenos que ocurren en los centros estudiados. Por este motivo, se utiliza un diseño descriptivo no experimental de carácter cuantitativo (García y Martínez, 2012).

Seguidamente pasamos a describir el procedimiento desarrollado. Tras solicitar los correspondientes permisos y la autorización de la Consejería de Educación, se realizó una primera comunicación con los equipos directivos de los centros seleccionados para comentar los objetivos de la investigación, solicitando la colaboración de los docentes y de las familias migrantes. Al profesorado se le envió el cuestionario mediante correo electrónico, y se llevó a cabo un seguimiento de este por medios telemáticos, con el fin de confirmar su recepción, cumplimentación y devolución. En cuanto a los progenitores, en una reunión se les expuso el contenido de los instrumentos, se les informó del cronograma previsto para la cumplimentación de los cuestionarios y para la devolución de los mismos, ofreciéndoles ayuda idiomática en todo momento a aquellos padres no hispano-hablantes. Para ambos colectivos, se garantizó el anonimato y la confidencialidad durante todo el proceso.

\section{Análisis de la información}

El análisis de los datos de carácter cuantitativo se ha realizado con el paquete estadístico Statistical Packa-ge for the Social Sciences (SPSS) versión 24, con el que se han llevado a cabo tanto los estadísticos descriptivos (media, desviación típica) como los inferenciales no paramétricos mediante la prueba $U$ de Mann-Whitney, adoptando el nivel de significación estadística $p \leq .05$. Ambos tipos de pruebas se han aplicado a los objetivos 1 y 3 , con la finalidad de realizar un análisis comparativo de dos grupos independientes sobre el conocimiento que 
poseen las familias migrantes de la vida del centro escolar e identificar el tipo de relaciones personales que mantienen dichos progenitores con el profesorado. Sin embargo, en el objetivo 2 se han aplicado únicamente las pruebas de estadísticos descriptivos, ya que no se compara la misma información planteada a los dos grupos participantes, sino que se trata de profundizar en el conocimiento que posee el profesorado sobre la vida del alumnado migrante.

\section{Resultados}

Objetivo 1. Analizar el conocimiento de la vida del centro que poseen las familias migrantes.

En la Tabla 1 se presentan los resultados referidos al conocimiento de la vida del centro educativo que poseen las familias migrantes, considerando las respuestas de los propios progenitores y del profesorado.

\section{Tabla 1}

Estadísticos descriptivos y significación estadística. Conocimiento de la vida del centro escolar que poseen las familias migrantes.

\begin{tabular}{|c|c|c|c|c|c|}
\hline ÍTEMS & $\begin{array}{l}\text { FAMILIA } \\
\text { PROFES. }\end{array}$ & CUESTIONES & M & Sd & $\begin{array}{c}\text { Significación } \\
\text { Estadística }\end{array}$ \\
\hline 1.1 & $\mathbf{F}$ & Conozco las Normas de Organización & 3.59 & 1.402 & .001 \\
\hline & $\mathbf{P}$ & $\begin{array}{l}\text { Funcionamiento del centro donde estudia mi hijo } \\
\text { Los padres de mis alumnos conocen las Normas de } \\
\text { Organización y Funcionamiento del centro }\end{array}$ & 3.34 & 1.029 & \\
\hline 1.2 & $\begin{array}{l}\mathbf{F} \\
\mathbf{P}\end{array}$ & $\begin{array}{l}\text { Conozco personalmente al tutor de mi hijo } \\
\text { Me conocen personalmente los padres de mis alumnos }\end{array}$ & $\begin{array}{l}4.25 \\
3.90\end{array}$ & $\begin{array}{c}1.035 \\
.925\end{array}$ & .000 \\
\hline 1.3 & $\mathbf{F}$ & $\begin{array}{l}\text { Conozco al resto de profesores que imparten clase a mi } \\
\text { hijo } \\
\text { Los padres de mis alumnos conocen al resto de } \\
\text { profesores de sus hijos }\end{array}$ & 3.88 & $\begin{array}{l}1.165 \\
.949\end{array}$ & .000 \\
\hline 1.4 & $\mathbf{F}$ & $\begin{array}{l}\text { Conozco la Propuesta Curricular Docente que utilizan } \\
\text { los profesores de mi hijo } \\
\text { Los padres de mis alumnos están informados sobre la } \\
\text { Propuesta Curricular Docente }\end{array}$ & 3.13 & 1.413 & .758 \\
\hline 1.5 & $\mathbf{F}$ & $\begin{array}{l}\text { Tengo información sobre el Plan de Recuperación y } \\
\text { Refuerzo que utiliza el centro educativo donde cursa mi } \\
\text { hijo } \\
\text { Los padres de mis alumnos conocen el Plan de } \\
\text { Recuperación y Refuerzo que utilizamos los profesores }\end{array}$ & 3.11 & 1.150 & .509 \\
\hline 1.6 & $\mathbf{F}$ & $\begin{array}{l}\text { Tengo información sobre el Plan de Convivencia del } \\
\text { centro donde cursa estudios mi hijo } \\
\text { Los padres de mis alumnos tienen información sobre el } \\
\text { Plan de Convivencia del Centro }\end{array}$ & 3.24 & $\begin{array}{l}1.436 \\
1.158\end{array}$ & .923 \\
\hline 1.7 & $\mathbf{P}$ & $\begin{array}{l}\text { Conozco las actividades de la Asociación de Madres y } \\
\text { Padres (AMPA) del centro } \\
\text { Los padres de mis alumnos conocen las actividades de } \\
\text { la Asociación de Madres y Padres (AMPA) del centro }\end{array}$ & 2.87 & $\begin{array}{l}1.502 \\
1.280\end{array}$ & .056 \\
\hline
\end{tabular}

Fuente: Elaboración propia 
Seguidamente se interpretan los resultados de mayores a menores puntuaciones medias. En lo que se refiere a si las familias conocen personalmente al tutor de sus hijos, los progenitores otorgan valores altos (ítem F.1.2; $\mathrm{M}=4.25$ ), y los docentes asignan puntuaciones próximas a altas (ítem P.1.2; $\mathrm{M}=3.90$ ). En cuanto a si los padres conocen al resto de profesores que imparten clase a sus hijos, las familias señalan valoraciones próximas a altas (ítem $F 1.3 ; M=3.88$ ), frente a puntuaciones moderadas en las respuestas del profesorado (ítem P.1.3; M=3.28).

En relación a si los progenitores poseen información sobre las normas de organización y funcionamiento del centro escolar, los padres señalan valores medio-altos (ítem F.1.1; M=3.59) y los docentes indican puntuaciones moderadas (ítem P.1.1; M=3.34).

Respecto a la significación estadística, tras aplicar la prueba $U$ de Mann Whitney, como se aprecia en la Tabla 1, en todos los ítems las diferencias entre los dos colectivos han resultado significativas excepto la referida: al ítem $1.6(p=.923)$ : Tengo información sobre el Plan de Convivencia del centro donde cursa estudios mi hijo; la referida al ítem 1.4 ( $p=.758)$ : Conozco la Propuesta Curricular Docente que utilizan los profesores de mi hijo; la referida al ítem 1.5 $(p=.509)$ : Tengo información sobre el Plan de Recuperación y Refuerzo que utiliza el centro educativo donde cursa mi hijo; la referida al ítem $1.7(p=.056)$ : Conozco las actividades de la Asociación de Madres y Padres (AMPA) del centro.

Objetivo 2. Describir el conocimiento que posee el profesorado sobre la vida del alumnado migrante.

En la Tabla 2 se detallan los estadísticos descriptivos referidos al conocimiento de la vida del alumno y alumna que posee el profesorado, según la opinión de los propios docentes.

\section{Tabla 2}

Estadísticos descriptivos (Media y desviación típica). Conocimiento de la vida del alumno y alumnas que posee el profesorado.

\begin{tabular}{|c|c|c|c|c|}
\hline ÍTEMS & $\begin{array}{l}\text { PROFE } \\
\text { S. }\end{array}$ & CUESTIONES & M & Sd \\
\hline 2.1 & $\mathbf{P}$ & Conozco a las madres de mis alumnos & 4.01 & .973 \\
\hline 2.2 & $\mathbf{P}$ & Conozco a los padres de mis alumnos & 2.94 & .957 \\
\hline 2.3 & $\mathbf{P}$ & $\begin{array}{l}\text { Estoy informado acerca de los miembros que componen la } \\
\text { unidad familiar de mis alumnos }\end{array}$ & 3.77 & .928 \\
\hline 2.4 & $\mathbf{P}$ & Conozco la profesión de los padres de mis alumnos & 3.31 & .948 \\
\hline 2.5 & $\mathbf{P}$ & $\begin{array}{l}\text { Conozco el grado y la forma en que los padres de mis } \\
\text { alumnos contribuyen al desarrollo de sus aprendizajes }\end{array}$ & 3.33 & .980 \\
\hline 2.6 & $\mathbf{P}$ & $\begin{array}{l}\text { Conozco qué alumnos viven fuera de la zona geográfica del } \\
\text { centro }\end{array}$ & 3.79 & 1.089 \\
\hline 2.7 & $\mathbf{P}$ & $\begin{array}{l}\text { Conozco el grupo de compañeros con los que suelen } \\
\text { relacionarse mis alumnos }\end{array}$ & 3.87 & 1.041 \\
\hline 2.8 & $\mathbf{P}$ & Conozco los problemas de aprendizaje de mis alumnos & 4.45 & 710 \\
\hline 2.9 & $\mathbf{P}$ & $\begin{array}{l}\text { Conozco los alumnos que tienen problemas psico-afectivos } \\
\text { relevantes }\end{array}$ & 4.64 & 3.238 \\
\hline 2.10 & $\mathbf{P}$ & $\begin{array}{l}\text { Estoy informado sobre posibles situaciones problemáticas } \\
\text { familiares de mis alumnos }\end{array}$ & 4.07 & .872 \\
\hline 2.11 & $\mathbf{P}$ & $\begin{array}{l}\text { Conozco a los alumnos que presentan problemas en sus } \\
\text { relaciones sociales }\end{array}$ & 4.22 & .826 \\
\hline 2.12 & $\mathbf{P}$ & $\begin{array}{l}\text { Tengo conocimiento sobre otros aspectos relevantes en la } \\
\text { vida de mis alumnos }\end{array}$ & 3.47 & 1.079 \\
\hline
\end{tabular}

Fuente: Elaboración propia 
En relación a si los docentes conocen los problemas de aprendizaje de los alumnos, los profesores indican en sus respuestas valores entre altos y muy altos (ítem P.2.8; M=4.45). En cuanto a si el profesorado conoce los problemas psico-afectivos relevantes que pueden presentar algunos alumnos, se constatan puntuaciones entre altas y muy altas (ítem P.2.9; M=4.64). En lo que se refiere a identificar a aquellos alumnos que presentan problemas en sus relaciones sociales, se contemplan valores altos (ítem P.2.11; $M=4.22$ ).

Respecto a si el profesorado está informado sobre posibles situaciones problemáticas familiares de los alumnos, se obtienen valores altos (ítem P.2.10; $M=4.07$ ). Cuando se les pregunta si conocen personalmente a las madres de los alumnos, se registran de nuevo puntuaciones altas (ítem P.2.1; $M=4.01$ ). En relación a si los docentes conocen a los padres, aparecen valores próximos a moderados (ítem P.2.2; $\mathrm{M}=2.94$ ).

En lo que se refiere al conocimiento que posee el profesorado sobre el grupo de compañeros con los que suelen relacionarse los alumnos (ítem P.2.7; M=3.87), los valores de sus respuestas son próximos a altos. En relación a si conocen a aquellos alumnos que residen fuera de la zona geográfica del centro, se obtienen nuevamente puntuaciones próximas a altas (ítem P.2.6; $\mathrm{M}=3.79$ ). Respecto a si están informados acerca de los miembros que componen la unidad familiar de los alumnos, aparecen valores próximos a altos (ítem P.2.3; M=3.77).

Los docentes están informados sobre otros aspectos relevantes de la vida de los alumnos, obteniendo puntuaciones medio-altas (ítem P.2.12; $\mathrm{M}=3.47$ ). En relación al grado y a la forma en que los padres contribuyen al desarrollo de los aprendizajes de los hijos, los profesores otorgan valores moderados (ítem P.2.5; M=3.33). Y, por último, si los docentes conocen la profesión de los padres de sus alumnos, se registran en sus respuestas valores nuevamente moderados (ítem P.2.4; $M=3.31$ ).

Objetivo 3. Identificar el tipo de relaciones personales que mantienen las familias migrantes y el profesorado.

En la Tabla 3 se exponen los resultados vinculados al tipo de relaciones que establecen tanto los padres migrantes como los docentes, considerando las respuestas de ambas partes.

\section{Tabla 3}

Estadísticos descriptivos y significación estadística. Tipo de relaciones que mantienen las familias migrantes con el profesorado.

\begin{tabular}{|c|c|c|c|c|c|}
\hline İTEMS & $\begin{array}{l}\text { FAMILIA } \\
\text { PROFES. }\end{array}$ & CUESTIONES & $\mathbf{M}$ & Sd & $\begin{array}{c}\text { Significación } \\
\text { Estadística }\end{array}$ \\
\hline \multirow[t]{2}{*}{3.1} & $F$ & $\begin{array}{l}\text { Asisto por propia iniciativa a visitar a los profesores de } \\
\text { mi hijo }\end{array}$ & 3.39 & 1.274 & .000 \\
\hline & $\mathbf{P}$ & $\begin{array}{l}\text { Los padres asisten por propia iniciativa a visitar a los } \\
\text { profesores de sus hijos }\end{array}$ & 2.30 & .995 & \\
\hline \multirow[t]{2}{*}{3.2} & $\mathbf{F}$ & $\begin{array}{l}\text { Los profesores de mi hijo facilitan que me sienta } \\
\text { cómodo y libre para expresar mis ideas }\end{array}$ & 4.04 & 1.095 & .000 \\
\hline & $\mathbf{P}$ & $\begin{array}{l}\text { Facilito a los padres de mis alumnos que se encuentren } \\
\text { cómodos y libres para expresar sus ideas }\end{array}$ & 4.65 & .581 & \\
\hline \multirow[t]{2}{*}{3.3} & $\mathbf{F}$ & $\begin{array}{l}\text { Tengo confianza con los profesores para hablar de mi } \\
\text { hijo }\end{array}$ & 4.18 & .994 & .000 \\
\hline & $\mathbf{P}$ & $\begin{array}{l}\text { Proporciono confianza a los padres de mis alumnos } \\
\text { cuando trato con ellos }\end{array}$ & 4.55 & .640 & \\
\hline
\end{tabular}

Fuente: Elaboración propia 
Tabla 3 (Continuación)

Estadísticos descriptivos y significación estadística. Tipo de relaciones que mantienen las familias migrantes con el profesorado.

\begin{tabular}{|c|c|c|c|c|c|}
\hline İTEMS & $\begin{array}{l}\text { FAMILIA } \\
\text { PROFES. }\end{array}$ & CUESTIONES & M & Sd & $\begin{array}{c}\text { Significación } \\
\text { Estadística }\end{array}$ \\
\hline \multirow[t]{2}{*}{3.4} & $F$ & $\begin{array}{l}\text { El profesorado admite las sugerencias de mejora } \\
\text { respecto a la educación de mi hijo, cuando se las } \\
\text { propongo }\end{array}$ & 3.97 & 1.067 & .000 \\
\hline & $\mathbf{P}$ & $\begin{array}{l}\text { Admito de los padres las sugerencias de mejora } \\
\text { respecto a la educación de su hijo, cuando me las } \\
\text { proponen }\end{array}$ & 4.37 & .719 & \\
\hline \multirow[t]{2}{*}{3.5} & $\mathbf{F}$ & $\begin{array}{l}\text { Recibo orientación y asesoramiento, por parte de los } \\
\text { profesores de mi hijo, en temas educativos }\end{array}$ & 3.84 & 1.212 & .000 \\
\hline & $\mathbf{P}$ & $\begin{array}{l}\text { Proporciono orientación y asesoramiento a los padres } \\
\text { de mis alumnos en temas educativos }\end{array}$ & 4.38 & .730 & \\
\hline \multirow[t]{2}{*}{3.6} & $\mathbf{F}$ & $\begin{array}{l}\text { He tenido dificultades o conflictos con algún profesor de } \\
\text { mi hijo }\end{array}$ & 1.54 & 1.130 & .000 \\
\hline & $\mathbf{P}$ & $\begin{array}{l}\text { He tenido dificultades o conflictos con algún padre de } \\
\text { mis alumnos }\end{array}$ & 1.97 & 1.108 & \\
\hline \multirow[t]{2}{*}{3.7} & $\mathbf{F}$ & $\begin{array}{l}\text { Cuando he querido contactar con algún profesor de mi } \\
\text { hijo, éste se ha mostrado accesible y dispuesto para la } \\
\text { comunicación }\end{array}$ & 4.06 & 1.199 & .000 \\
\hline & $\mathbf{P}$ & $\begin{array}{l}\text { Me muestro accesible y dispuesto para la comunicación } \\
\text { y colaboración con los padres }\end{array}$ & 4.60 & .624 & \\
\hline
\end{tabular}

Fuente: Elaboración propia

En lo que respecta a si las familias que tienen confianza con los profesores para hablar de los hijos, los progenitores asignan valores altos (ítem F.3.3; $M=4.18$ ), y los docentes otorgan puntuaciones entre altas y muy altas (ítem P.3.3; $\mathrm{M}=4.55$ ). En cuanto a si los progenitores han querido contactar con algún profesor de sus hijos, las familias indican valores altos (ítem F.3.7; $M=4.06$ ) y los docentes señalan en sus respuestas puntuaciones entre altas y muy altas (ítem P.3.7; $M=4.60)$.

En lo que se refiere a si los profesores facilitan que los padres se sientan cómodos y libres para expresar sus ideas, los progenitores señalan valores altos (ítem F.3.2; M=4.04), y el profesorado asigna puntuaciones entre altas y muy altas (ítem P.3.2; $M=4.65$ ). En relación a si el profesorado admite las sugerencias de mejora, respecto a la educación de los alumnos, las familias proporcionan valores próximos a altos (ítem F.3.4; $\mathrm{M}=3.97$ ) y el profesorado otorga puntuaciones altas (ítem P.3.4; M=4.37).

Respecto a si los progenitores reciben orientación y asesoramiento, por parte de los profesores de los hijos, en temas educativos, los padres indican valores próximos a altos (ítem F.3.5; $M=3.84$ ) y los docentes otorgan puntuaciones altas al respecto (ítem P.3.5; $M=4.38$ ). En cuanto a la asistencia por propia iniciativa de los padres a visitar a los docentes, las familias indican valores moderados (ítem F.3.1; M=3.39), mientras que los profesores muestran puntuaciones bajas (ítem P.3.1; $M=2.30$ ). Finalmente, en relación a si ambas partes han tenido algunas dificultades o conflictos, los progenitores indican valores entre bajos y muy bajos (ítem F.3.6; $M=1.54$ ), y los docentes señalan puntuaciones próximas a bajas (ítem P.3.6; $M=1.97$ ).

En lo que se refiere a la significación estadística, tras aplicar la prueba $U$ de Mann Whitney, como se observa en la Tabla 3, en todos los ítems las diferencias entre los dos colectivos han resultado significativas. 


\section{Discusión y Conclusiones}

A la luz de los resultados expuestos en el apartado anterior, se constata que el dominio de los progenitores sobre las normas de organización y funcionamiento del centro escolar es bastante bueno, aunque el profesorado considera que no lo es tanto, sino que más bien es moderado. Datos similares aparecen en la investigación desarrollada por Llevot y Bernad (2015), cuando analizan el manejo que tienen las familias sobre la organización y el funcionamiento de los centros educativos. El estudio revela que las familias suelen acudir al centro cuando son convocadas a una reunión colectiva a principios del curso escolar para informarles sobre la organización del centro; pero dicha presencia disminuye conforme avanza el año académico. González (2007) considera que es insuficiente el conocimiento que muchas familias migrantes tienen respecto al funcionamiento y organización de la institución escolar, lo que se refleja en las escasas visitas que realizan los progenitores al centro educativo. Si hay algo en lo que claramente coinciden las distintas investigaciones es en el escaso conocimiento que las familias migrantes tienen sobre las normas educativas, a nivel general, y de la cultura escolar de acogida, a nivel particular.

Las familias conocen bien a los profesores tutores de sus hijos, al igual que al resto de los profesores que les imparten clase. Sin embargo, los docentes tienen un conocimiento moderado de los progenitores del alumnado migrante. Similares opiniones muestran otras investigaciones que inciden en el mayor conocimiento que tienen los padres migrantes del profesorado y viceversa (González et al., 2016), lo que nos hace reflexionar acerca de las opiniones que sobre ello muchas veces manifiesta el profesorado.

No existen diferencias de opinión respecto a la información que tienen los padres sobre: propuesta curricular docente, el plan de recuperación y refuerzo, y el plan de convivencia. El estudio llevado a cabo por Hernández Prados, et al. (2016) coincide con los hallazgos de nuestro estudio y añade que el conocimiento de los progenitores migrantes sobre dichos aspectos es mucho más limitado.

Se observa que las familias migrantes no tienen un gran conocimiento sobre las actividades que se llevan a cabo desde la Asociación de Madres y Padres (AMPA). En tal sentido, la investigación de González (2007), que analiza las necesidades de orientación y formación que los padres y las madres migrantes suelen presentar a la hora de participar en los centros educativos, destaca el escaso conocimiento que las familias migrantes tienen sobre la cultura escolar de acogida. Tampoco conocen las actividades que organiza la AMPA.

Cabe destacar que los profesores conocen personalmente a las madres de sus alumnos, pero no tanto a los padres. Tanto los padres como las madres migrantes deben ser conscientes de que la calidad de la educación comienza con la contribución e implicación de ambos progenitores de manera activa en el centro educativo. En relación a si el profesorado está informado de los miembros que componen la unidad familiar, se ha comprobado que está bien informado, aunque conoce poco la actividad laboral de los padres de sus alumnos.

Los progenitores no contribuyen excesivamente al desarrollo de los aprendizajes de sus hijos según la información de nuestra investigación. Estos resultados no coinciden con los obtenidos por García Sanz et al., (2010), que sostienen que los padres contribuyen bastante al aprendizaje de los hijos, y que a menudo están dispuestos a supervisar y controlar su evolución, así como a analizar cualquier otra actividad al objeto de mejorar este proceso. Se deduce de ello que el grado de contribución de las familias puede facilitar o dificultar el proceso educativo de los hijos, no siendo el mismo en todas las ocasiones (Gomáriz et al., 2019; Rubie-Davies et al., 2010). 
Los profesores conocen bastante bien a los alumnos que viven fuera de la zona geográfica del centro, lo cual es muy importante ya que, como indica Leiva (2013b), es fundamental que el profesorado comprenda el contexto social donde se desenvuelve la vida de las familias migrantes. Se ha comprobado asimismo que los docentes tienen buena información sobre el grupo de compañeros con los que suelen relacionarse sus alumnos, lo que consideramos muy importante. Es determinante, por consiguiente, que los docentes conozcan las relaciones sociales que establece el alumnado respecto a su grupo de iguales, ya que numerosos alumnos y alumnas migrantes mantienen más contacto con compañeros del mismo país de origen, que con amistades de compañeros de origen autóctono (Chamseddine, 2018; Intxausti et al., 2014), lo que limita su círculo de amistades y dificulta que se establezcan vínculos interculturales y de respeto entre las diferentes culturas.

Se ha puesto en evidencia que los docentes identifican bastante bien los problemas de aprendizaje de sus alumnos. Jiménez (2007) expresa y resalta el seguimiento exhaustivo que el profesorado realiza sobre las diferentes dificultades que presenta la diversidad de discentes en las aulas. De igual forma, el profesorado reconoce a los alumnos que tienen problemas personales y psico-afectivos relevantes, que afectan a su rendimiento académico y a sus relaciones sociales (Aguado, et al, 2003; Gómez Jarabo, 2015; Rodríguez y Fernández, 2018). Portes y Rumbaut (2001) y Terrén y Carrasco (2007) manifiestan el gran deseo de las familias por ayudar a sus hijos a superar las posibles dificultades que se producen en las relaciones sociales, y las altas expectativas que tienen acerca de este particular. Por todo ello, cabe destacar la necesidad de que se tengan en cuenta todos estos factores en las aulas, de manera que aumenten y se rentabilicen las relaciones interpersonales entre todo el alumnado, lo que redundaría en ayuda mutua y contribuiría a la mejora de los contextos multiculturales en los que viven y al desarrollo de una educación inclusiva e intercultural (Arnaiz, 2019).

En relación a si los docentes están informados sobre posibles situaciones problemáticas familiares de sus alumnos, se confirma que sí lo están, lo cual tiene una gran importancia para sus aprendizajes. Datos similares aparecen en el estudio llevado a cabo por Wood et al. (2007), que revela que el alto interés de los docentes sobre las posibles situaciones problemáticas de sus alumnos, repercute en gran medida en su proceso formativo. Benner y Mistry (2007) concluyen que, cuando existen bajas expectativas del profesorado, en relación a la resolución de los problemas familiares, se estimulan de manera sistemática las expectativas familiares y estas contribuyen, a su vez, a mitigar el impacto negativo en el alumnado.

Las visitas de las familias migrantes a los centros, por iniciativa propia, aparece como una tarea pendiente todavía en los centros. Los trabajos de Martínez González y Pérez Herrero (2006) apuntan en la misma dirección; indican que la visita de los padres a las escuelas por iniciativa propia sólo se produce en caso de incidencias o para abordar los temas de las calificaciones de sus hijos como manifiesta Megías Quirós (2006). No obstante, los progenitores se sienten cómodos cuando asisten a las reuniones y manifiestan que los profesores facilitan su acogida y estancia en el centro, favoreciendo que expresen sus ideas. Esta información es corroborada por los docentes.

En la investigación desarrollada por Hampden y Galindo (2016) se indica que una buena relación entre familia y escuela es muy positiva para los docentes y bastante beneficiosa para los progenitores, especialmente, cuando es una relación basada en una confianza mutua. Estos resultados son similares a los obtenidos en el estudio de Loudová (2013) donde también se han encontrado estos mismos resultados. Por el contrario, la investigación llevada a cabo por Llevot y Bernad (2015) constata que la confianza entre ambas partes podría mejorar ya que no alcanza los objetivos deseados por la comunidad educativa. Andrés y Giró (2016) sostienen que conseguir la confianza plena entre ambos colectivos implica mostrar y compartir el mismo mensaje, y la misma visión acerca de la educación, puesto que si esta es disonante o contrapuesta repercutirá negativamente en todo el proceso educativo del alumnado. 
En lo que se refiere a si el profesorado admite las sugerencias de mejora respecto a la educación del alumnado migrante, las familias opinan que aceptan sus propuestas. Por su parte, el profesorado manifiesta que siempre admite las sugerencias de los padres. En cambio, en el estudio realizado por Vallespir et al. (2016) se expone que existen algunas dificultades por parte de las familias migrantes para aceptar las sugerencias de los docentes, ya que les cuesta trabajo mantener una relación fluida con agentes externos a la escuela. Respecto a si los progenitores reciben orientación y asesoramiento por parte de los profesores en temas educativos, se obtienen opiniones bastantes satisfactorias por parte de los padres, al considerarlo de vital importancia para la educación de sus hijos. Esta respuesta positiva de familias migrantes también es muy bien valorada por parte de los docentes. Otros autores, como Charette y Kalubi (2016) y Fagan (2013) matizan que, a pesar de la existencia de diferencias entre ambos colectivos en otros aspectos, tanto la orientación como el asesoramiento a los progenitores en aspectos educativos que afectan a sus hijos son muy bien acogidos y valorados.

Para finalizar, cabe señalar la inexistencia de dificultades o conflictos entre padres y docentes, lo que es corroborado por ambas partes. En general, el profesorado se muestra accesible y dispuesto a comunicarse con los padres de los alumnos migrantes cuando lo han requerido. Esta percepción es más alta en el profesorado que en las familias. No obstante, estas manifiestan que el profesorado siempre las ha atendido. Opiniones que difieren de las manifestadas por Rey Mantilla (2006), que ha encontrado una actitud cerrada de los docentes, fruto de la relación asimétrica de una buena parte de ellos, que obstaculiza la accesibilidad de las familias migrantes a los centros educativos.

A modo de conclusión, cabe indicar que las familias migrantes deben conocer mejor las normas de organización y funcionamiento del centro educativo al que asisten sus hijos. Aunque conocen personalmente al tutor de sus hijos y al resto de los profesores, necesitan adquirir un mayor conocimiento del plan de convivencia, de la propuesta curricular docente, del plan de recuperación y de refuerzo, así como de las actividades de la asociación de madres y padres para que su relación con el profesorado y la escuela sea más fluida.

Los docentes tienen un buen conocimiento de las dificultades que pueden presentar sus alumnos en las relaciones sociales, sus problemas de aprendizaje, psico-afectivos, y las posibles situaciones problemáticas en el seno familiar. En cambio, se constata que no conocen personalmente a los padres varones de sus alumnos, lo que confirma que la implicación de estos en la escuela sigue siendo escasa, en comparación con la de las madres, cuyo contacto con el centro escolar es mucho más regular. De igual forma deben ampliar su conocimiento acerca de los miembros que componen la unidad familiar, la profesión de ambos progenitores, su contribución en el aprendizaje de sus hijos, la zona geográfica donde residen, el grupo de compañeros con los que se suele relacionar el alumnado, y los aspectos relevantes de sus vidas.

Por tanto, se considera acuciante que el profesorado conozca tanto a las madres como a los padres de los discentes, teniendo en cuenta el valor de la responsabilidad compartida de ambos progenitores en la educación de sus hijos. También es imprescindible que se interese por la vida del alumnado de las familias migrantes, ya que muchas veces conocer el impacto de los condicionantes externos a la escuela que viven, puede facilitar u obstaculizar el proceso de enseñanza-aprendizaje de los escolares y, por consiguiente, el éxito académico de los mismos.

Finalmente, es preciso resaltar que las visitas de las familias migrantes al centro o la demanda de entrevistas al profesorado, por iniciativa propia, sigue siendo una asignatura pendiente. A pesar de avances relevantes, queda mucho por hacer. Es fundamental a este respecto el rol que desempeñen los docentes, ya que debe facilitar que las familias se sientan cómodas y libres para expresar sus ideas, brindarles confianza ante las situaciones de mejora, proporcionarles asesoramiento, así como mostrarles accesibilidad y predisposición para la comunicación. Consideramos necesario, pues, que se reflexione acerca de las percepciones que tiene el profesorado y las propias familias migrantes sobre el tipo de relaciones personales que deberían mantener. 
Es fundamental que los centros educativos, de atención preferente al alumnado vulnerable, implementen procesos de comunicación viables y alejados del modelo tradicional etnocentrista, instalado en estereotipos culturales y sociales que aún hoy en día coexisten en el contexto escolar. Todo ello alimenta el rechazo y el recelo, e incrementa el trato hostil y distante, impidiendo una interacción fluida entre ambas partes. Dichos comportamientos, reales y observables en numerosos centros educativos, en nuestra opinión, obstaculizan la oportunidad de un buen conocimiento e intercambio. Por tanto, afecta a la confianza mutua, como motor esencial para una relación estable y de calidad.

En definitiva, promover la orientación de los progenitores migrantes, en cuanto a su conocimiento de la escuela y su relación con el profesorado, posibilitaría abordar de manera adecuada el proceso educativo de sus hijos, debiendo formar parte de las prioridades de los centros educativos. Se trata, por consiguiente, de diseñar estrategias y metodologías inclusivas acordes con las peculiaridades, intereses y expectativas de las familias migrantes, tantas veces en situación de vulnerabilidad, que garanticen para ellas los principios de igualdad, equidad e inclusión social y educativa.

La limitación de esta investigación estriba en la necesidad de analizar otras variables que posibiliten conocer mejor el funcionamiento de estos centros de atención preferente para alumnado vulnerable y su relación con las familias migrantes. Entre ellas destacamos buscar diferencias en los resultados según el origen y el nivel social de las familias migrantes y el idioma materno. En cuanto a los docentes cabría estudiar variables tales como su experiencia docente, género y formación. También sería de interés llevar a cabo entrevistas a las familias o grupos focales con el profesorado, para conocer con mayor profundidad todo lo aquí tratado, lo que indudablemente completaría el análisis realizado.

\section{Referencias bibliográficas}

Aguado, T., Ballesteros, B., Malik, B. y Sánchez, M. (2003). Educación intercultural en la enseñanza obligatoria: prácticas escolares; actitudes y opiniones de padres, alumnos y profesores; resultados académicos de los estudiantes de diversos grupos culturales. Revista de Investigación Educativa, 21(2), 323-348. https://dialnet.unirioja.es/

Andrés Cabello, S. y Giró Miranda, G. (2016). La participación de las familias en la escuela: una cuestión compleja. Revista de Evaluación de Programas y Políticas Públicas, 7, 28-47. https://doi.org/10.5944/reppp.7.2016.16302

Aparicio, R. (Coord.) (2003). Red de menores extranjeros escolarizados. La familia y la integración escolar de los menores de origen extranjero. Instituto Universitario de Estudios sobre Migraciones.

Arnaiz Sánchez, P. (2019). La educación inclusiva: Mejora escolar y retos para el siglo XXI. Participación Educativa, 9,195-207. https://www.educacionyfp.gob.es/dam/jcr:559207c72901-4d6e-82a3-b016d74adaa3/pe-n9-art03-pilar-arnaiz.pdf

Benner, A. D. y Mistry, R. S. (2007). Congruence of mother and teacher educational expectations and low-income youth's academic competence. Journal of Educational Psychology, 99(1), 140-153. https://doi.org/10.1037/ 00220663.99.1.140.

Ceballos López, N. y Saiz Linares, A. (2019). La acción tutorial como escenario de colaboración familia-escuela. REOP, 30(2), 28-45. https://doi.org/10.5944/reop.vol.30.num.2.2019.25336 
chamseddine Habib Allah, m. (2018). Polarización escolar en España. Retos e implicaciones International Journal of Educational Research and Innovation (IJERI), 9, 189-205.

chamseddine Habib Allah, m. (2020). Study on communication between migrant families and schools. Revista Electrónica Interuniversitaria de Formación del Profesorado, 23(1), 33-47. https://doi.org/10.6018/reifop.404181

chamseddine Habib Allah, M, y Hernández Martín, A. (2020). Actitudes de los escolares ante la llegada de población migrante: Estudio comparativo y aproximación al desarrollo de buenas prácticas educativas. Revista Educatio Siglo XXI, 38(1), 229-252. http://dx.doi.org/10.6018/educatio.413491

Charette, J. y Kalubi, J.C. (2016). Collaborations école-famille-communauté: I'apport de I'intervenant interculturel dans l'accompagnement à l'école de parents récemment immigrés au Québec. Education Sciences \& Society, 2, 127-149. http://tcri.qc.ca/images/publications/volets/volet-jeunes/2017/Actes_RN_ICI_ENJEUmai_2017-Documents-internes/Josee_Charette_article_2.pdf

Dubet, F. (DIR.) (1997). École, Familles. Le malentendu. Textuel.

Fagan, P.F. (2013). Family and Education. Revista Estudios sobre Educación, 25, 167-186. https://hdl.handle.net/10171/34775

Garcia, M.P. y Martínez, P. (2012). Guía práctica para la realización de trabajos Fin de Grado y trabajos Fin de Master. Editum.

García Sanz, M.P., Gomariz Vicente, M.A., Hernández Prados, M.A. y Parra Martínez, J. (2010). La comunicación entre la familia y el centro educativo, desde la percepción de los padres y madres de los alumnos. Educatio Siglo XXI, 28(1), 157-188. https://revistas.um.es/educatio/article/view/109771

Garreta, J. y Llevot, N. (2007). La relación familia-escuela: ¿una cuestión pendiente? En J. Garreta (ed.), La Relación familia-escuela (pp. 9-12). Edicions de la Universitat de Lleida.

Gigli, A., Demozzi, D. y Pina-Castillo, M. (2019). La alianza educativa escuela/familia y los grupos de chat de padres: una mirada a la situación italiana. Revista Electrónica Interuniversitaria de Formación del Profesorado, 22(3), 15-30. https://doi.org/10.6018/reifop.389271

Gomariz, M.A., Parra, J., García Sanz, M.P., Hernández Prados, M.A. y Pérez Cobacho, J. (2008). La comunicación entre la familia y el centro educativo. Consejo Escolar de la Región de Murcia. Consejería de Educación, Ciencia e Investigación.

Gomariz Vicente, M.A., Martínez-Segura, M.J. y Parra Martínez, J. (2019). Desde la implicación en el hogar de las familias a la facilitación de los docentes en un contexto multicultural. Revista Electrónica Interuniversitaria de Formación del Profesorado, 22(3), 45-60. https://doi.org/10.6018/reifop.389631

Gómez Jarabo, I. (2015). Formación del profesorado para el tratamiento educativo de los conflictos sobre diversidad cultural y de género. (Tesis Doctoral). Universidad Complutense de Madrid.

González Falcón, I. (2007). La participación de las familias inmigrantes en la escuela: necesidades de orientación y formación. Revista de Educación, 9, 155-169. https://doi.org/10.7203/relieve.23.1.9258

Hampden Thompson, G. y Galindo, C. (2016). School-family relationships, school satisfaction and the academic achievement of young people. Educational Review, 69(2), 248-265. https://doi.org/10.1080/00131911.2016.1207613 
Hernández Prados, M.A. y Alcántara Colomer, M.M. (2016). La participación familiar de la población inmigrante en la escuela. Revista Familia, 52, 41-61. https://dialnet.unirioja.es/servlet/articulo?codigo $=5372834$

Hernández Prados, M.A., Gomariz Vicente M.A., Parra Martínez, J. y García Sanz, M.P. (2016). Familia, inmigración y comunicación con el centro escolar: un estudio comparativo. Revista Educación XX1. 19(2), 127-151. https://doi.org/10.5944/ educXX1.16457

Intxausti, N., Etxeberria, F. y Joaristi, L. (2014). ¿Coinciden las expectativas escolares de la familia y del profesorado acerca del alumnado de origen inmigrante? RELIEVE, 20(1), 1-19. https://doe.org/10.7203/relieve.20.1.3804.

Jiménez P. (2007). ¿Qué opinan los padres de niños con necesidades educativas especiales, inmigrantes y gitanos sobre la escuela de sus hijos? Bordón, 59(1), 95-111. https://recyt.fecyt.es/index.php/BORDON/article/view/36839

Leiva, J.J. (2013a). Bases conceptuales de la educación intercultural. De la diversidad cultural a la cultura de la diversidad. Revista Foro de Educación, 11(5), 169-197. http://dx.doi.org/10.14516/fde.2013.011.015.008

Leiva, J.J. (2013b). Relaciones interpersonales en contextos de Educación Intercultural: un estudio cualitativo. Dedica. Revista de Educação e Humanidades, 4, 109-128. https://dialnet.unirioja.es/ $=4252334$.

Llevot, N. y Bernad, O. (2015). La participación de las familias en la escuela: Factores claves. Revista de la Asociación de Sociología de la Educación, 8(1), 57-70. https://ojs.uv.es/index.php/RASE/article/view/8761/8304

Loudová, I. (2013). Communication of school and family in relation to the form teacher. ProcediaSocial and Behavioral Sciences, 651-657. https://doi.org/10.1016/j.sbspro.2013.12.074

Macía Bordalba, M. (2019). Principales canales para la comunicación familia-escuela: análisis de necesidades y propuestas de mejora. Revista Complutense de Educación, 30(1), 147-165. https://doi.org/10.5209/RCED.56034

Madrid, R., Saracostti, M., Reininger, T. y Hernández, M.T. (2019). Responsabilización, obediencia y resistencia: perspectivas de docentes y padres sobre la colaboración familia-escuela. Revista Electrónica Interuniversitaria de Formación del Profesorado, 22(3), 1-13. http://dx.doi.org/10.6018/reifop.22.3.389801

Martínez González, R.A. y Pérez Herrero, M. H. (2006). Propuestas metodológicas para una educación de calidad a través de las relaciones entre centros docentes, familias y entidades comunitarias. Cultura y Educación, 18(3-4), 231-246. https://doi.org/10.1174/113564006779172957

Megías Quirós, I. (2006). Padres-docentes en la encrucijada educativa de los más pequeños. En M. I. Álvarez Vélez Y A. Berástegui Pedro-Viejo (coords.), Educación y familia: la educación familiar en un mundo en cambio (pp. 151-169). Universidad Pontificia Comillas.

Portes, A. y Rumbaut, R.G. (2001). Legacies. The story of the immigrant second generation. Russell Sage Foundation.

Rey Mantilla, R. (2006). La relación de la familia y el colegio. En M. I. Álvarez Vélez Y A. Berástegui Pedro-Viejo (coords.), Educación y familia: la educación familiar en un mundo en cambio (pp.139-150). Universidad Pontificia Comillas. 
Rodríguez Fuentes, A. y Fernández, A.D. (2018). Actitudes ante la diversidad cultural de progenitores y descendientes. Eficiencia de la influencia por su grado de confluencia. Arbor, 194, 1-15. https://doi.org/10.3989/arbor.2018.788n2011

Roth Eichin, N. y Volante Beach, P. (2018). Liderando alianzas entre escuelas, familias y comunidades: una revisión sistemática. Revista Complutense de Educación, 29(2), 595611. https://doi.org/10.5209/RCED.53526

Rubie-Davies, M., Peterson, E., Irving, E., Widdowson, D. y Dixon, R. (2010). Expectations of achievement. Student, teacher and parent perceptions. Research in Education, 83, 36-53. https://doi.org/10.7227/RIE.83.4

Santos-Rego, M.A., Lorenzo-Moledo, M. y Priegue-Caamano, D. (2019). La Mejora de la Participación e Implicación de las Familias en la Escuela: un Programa en acción. Revista Electrónica Interuniversitaria de Formación del Profesorado, 22(3), 93-107. http://dx.doi.org/10.6018/reifop.22.3.389931

Terrén, E. y Carrasco, C. (2007). Familia, escuela e inmigración. Migraciones, 22, 9-46.

Vallespir, J., Rincón, J.C. y Morey, M. (2016). La participación de las familias en el Consejo Escolar y la formación del profesorado. Revista Electrónica Interuniversitaria de Formación del Profesorado, 19(1), 31-45. http://dx.doi.org/10.6018/ reifop.19.1.245751.

Wood, D., Kaplan, R. y Mcloyd, V. C. (2007). Gender differences in the Educational Expectations of Urban, Low-Income African American Youth: The Role of Parents and the School. Journal of Youth Adolescence, 36, 417-427. https://doi.org/10.1007/s10964-007-9186-2.

Fecha de entrada: 23 diciembre 2019

Fecha de revisión: 29 mayo 2020

Fecha de aceptación: 4 junio 2020 